\author{
C. A. Curat $\cdot$ V. Wegner $\cdot$ C. Sengenès $\cdot$ A. Miranville $\cdot$ \\ C. Tonus $\cdot$ R. Busse $\cdot$ A. Bouloumié
}

\title{
Macrophages in human visceral adipose tissue: increased accumulation in obesity and a source of resistin and visfatin
}

Received: 6 October 2005 / Accepted: 25 November 2005 / Published online: 23 February 2006

(C) Springer-Verlag 2006

\begin{abstract}
Aims/hypothesis: Increased visceral white adipose tissue (WAT) is linked to the risk of developing diabetes. Methods/results: We showed by fluorescence activated cell sorting analysis that human visceral WAT contains macrophages, the proportion of which increased with obesity. Selective isolation of mature adipocytes and macrophages from human visceral WAT by CD14 immunoselection revealed that macrophages expressed higher levels of chemokines (monocyte chemotactic protein 1, macrophage inflammatory protein $1 \alpha$, IL-8) and the adipokines resistin and visfatin than did mature adipocytes, as assessed by real-time PCR analysis. Moreover, resistin and visfatin proteins were found to be released predominantly by visceral WAT macrophages. Macrophage-derived secretory products stimulated phosphorylation of protein kinase B in human hepatocytes. Conclusions/interpretation: Resistin and visfatin might be considered to be proinflammatory markers. The increased macrophage population in obese human visceral WAT might be responsible for the enhanced production of chemokines as well as resistin and visfatin.
\end{abstract}

C. A. Curat $\cdot$ V. Wegner $\cdot$ C. Sengenès $\cdot$ A. Miranville $\cdot$

R. Busse · A. Bouloumié $(\square)$

Institute of Cardiovascular Physiology,

Johann Wolfgang Goethe University,

Theodor-Stern Kai 7,

60590 Frankfurt am Main,

Germany

e-mail: Bouloumie@zphys1.uni-frankfurt.de

Tel.: +49-69-630183569

Fax: +49-69-63017668

C. Tonus

Department of Surgery I, Klinikum Offenbach,

Offenbach, Germany

\section{A. Bouloumié}

Obesity Research Unit, INSERM U 586,

Louis Bugnard Institute,

Centre Hospitalier Universitaire Rangueil,

Université Paul Sabatier,

Toulouse, France
Keywords Adipocytes - Adipokines - Akt . AMP-activated protein kinase - Chemokines - Cytokines . Inflammation · Interleukins · Liver · MAP kinases

Abbreviations Akt: protein kinase B - FACS: fluorescence activated cell sorting $\cdot \mathrm{MCP}-1$ : monocyte chemotactic protein 1 (CCL2) - MIP-1 $\alpha$ : macrophage inflammatory protein $1 \propto(\mathrm{CCL} 3) \cdot \mathrm{SVF}$ : stroma-vascular fraction - WAT: white adipose tissue

\section{Introduction}

Increased accumulation of white adipose tissue (WAT) in the viscera is considered a strong and independent predictor of adverse health outcomes associated with obesity. Visceral WAT, characterised by high metabolic turnover, is thought to provide increased NEFA to the liver via direct venous drainage, contributing to abnormalities in glucose homeostasis [1]. Besides its role in energy storage, WAT is now recognised as an active secretory organ through its production of adipokines. Adipokines have been involved in the modulation of glucose and lipid homeostasis via central effects of leptin or the peripheral actions of resistin, adiponectin and visfatin on the liver and muscle. In addition, adipokines include proinflammatory factors and chemokines, the production of which has been shown to be increased in obesity [2]. Recently, obesity has been associated with increased accumulation of macrophages within the fat mass in rodents [3] and subcutaneous WAT [4]. The present study was undertaken to characterise the macrophage population present in the human visceral fat mass.

\section{Subjects and methods}

Isolation of the stroma-vascular fraction, adipocytes and $\mathrm{CD} 14^{+}$cells from human visceral WAT

Human visceral WAT was obtained from patients (33 men, mean BMI $25.5 \pm 0.7 \mathrm{~kg} / \mathrm{m}^{2}$, age range $21-78$ years; 
17 women, mean BMI $25.4 \pm 1.4 \mathrm{~kg} / \mathrm{m}^{2}$, age range $43-$ 81 years) without known infection who were undergoing abdominal surgery $(80 \%$ gastrointestinal tumour resection). The study was approved by the ethical committee of the University Hospital, Frankfurt am Main. The use of human material conforms to the principles outlined in the Declaration of Helsinki.

Adipocytes and $\mathrm{CD} 14^{+}$cells from the stroma-vascular fraction (SVF) were isolated by immunoselection as described previously [4]. Fluorescence activated cell sorting (FACS) analyses were performed on freshly removed SVF and CD14 ${ }^{+}$cells using CD14-phycoerythrin, CD45-peridinin-chlorophyll-protein complex and CD31fluorescein isothiocyanate antibodies or isotype controls. The labelled cells were analysed by flow cytometry using a FACSCalibur flow cytometer (BD Biosciences, Heidelberg, Germany) and CellQuest Pro software (BD Biosciences). The concentrations of resistin and visfatin were determined by ELISAs from R\&D Systems (Wiesbaden, Germany) and Phoenix Europe (Karlsruhe, Germany), respectively.

RNA extraction and real-time PCR

Total RNA was extracted from freshly removed cells using RNeasy kit (Qiagen, Hilden, Germany). RNA $(1 \mu \mathrm{g})$ was reverse-transcribed using the SuperScript III RNase H RT system (Invitrogen, Karlsruhe, Germany). Taqman probes for IL-6, TNF- $\alpha$, adiponectin, visfatin and resistin were provided by Applied Biosystems (Assays-on-Demand: Hs00174131, Hs00236874, Hs00605917, Hs00237184 and Hs00220767, respectively; Applied Biosystems, Darmstadt, Germany). SYBR Green primers for the macrophage inflammatory protein $1 \propto$ (MIP- $1 \alpha$ ), monocyte chemotactic protein 1 (MCP-1), IL-8 and leptin were designed using Primer Express software (Applied Biosystems) (Table 1). The PCR mixtures were prepared with SYBR Green and TaqMan Universal PCR Master Mixes (Applied Biosystems). All amplification reactions were performed in duplicate from $20 \mathrm{ng}$ cDNA using the Mx4000 Multiplex Quantitative PCR System (Stratagene, La Jolla, CA, USA) using the following conditions: $50^{\circ} \mathrm{C}$ for $2 \mathrm{~min}, 95^{\circ} \mathrm{C}$ for $10 \mathrm{~min}$, followed by 40 cycles at $95^{\circ} \mathrm{C}$ for $15 \mathrm{~s}$ and $60^{\circ} \mathrm{C}$ for $1 \mathrm{~min}$. Results were analysed with Stratagene Mx4000 software and all values were normalised to the levels of the ribosomal RNAs.
Human hepatocyte culture and western blot analysis

Human Caucasian hepatocyte carcinoma cells (HepG2 cells) from the European Collection of Cell Cultures (Sigma, Munich, Germany) were seeded at $2-3 \times 10,000$ cells $/ \mathrm{cm}^{2}$ in $2 \mathrm{mmol} / 1 \mathrm{~L}$-glutamine, $1 \%$ non-essential amino acids, $10 \%$ fetal bovine serum in MEM medium. After serum deprivation for $24 \mathrm{~h}$, cells were treated for $3 \mathrm{~min}$ with conditioned media and lysed on ice for $20 \mathrm{~min}$ in lysis buffer. Cell lysate $(40 \mu \mathrm{g})$ was separated by SDS-PAGE under denaturing conditions and transferred to nitrocellulose membranes. After blocking, membranes were incubated overnight with antibodies. Immune complexes were visualised using chemiluminescence solutions. For reprobing, membranes were stripped in $70 \mathrm{mmol} / \mathrm{l}$ Tris- $\mathrm{HCl}(\mathrm{pH}$ 6.8), $2 \%$ SDS, $0.1 \% \quad \beta$-mercaptoethanol at $50^{\circ} \mathrm{C}$ for $15 \mathrm{~min}$. The autoradiographs were scanned with an imaging densitometer and quantified using Tina software (Raytest, Straubenhardt, Germany).

\section{Data analysis}

Values are given as mean \pm SEM of $n$ independent experiments. Correlations were analysed using Spearman's correlation test. Statistical significance was determined using Kruskal-Wallis analysis and the non-parametric Mann-Whitney test. A $p$ value $\leq 0.05$ was taken to indicate statistical significance.

\section{Results}

Increased macrophage accumulation in the human visceral WAT in obesity

FACS analyses, performed on the freshly removed SVF from human visceral WAT using an antibody directed against the monocyte/macrophage cell surface marker CD14, showed an increasing percentage of $\mathrm{CD} 14^{+}$cells in visceral WAT with increasing BMI (Fig. 1a). The CD14 population was positive in FACS analyses for the common leucocyte marker CD45 and the endothelial cell and monocyte/macrophage marker CD31 (data not shown).

Table 1 Sequences of the primers

\begin{tabular}{lll}
\hline & Forward & Reverse \\
\hline hMIP-1 $\alpha$ & TTTGCTCTGAGAGTTCCCCTG & AGCCACTCGGTTGTCACCA \\
hMCP-1 & CGCCTCCAGCATGAAAGTCT & ATGAAGGTGGCTGCTATG \\
hIL-8 & ACCGGAAGGAACCATCTCACT & GGAAGGCTGCCAAGAGAGC \\
hLeptin & CCTTCCAGAAACGTGATCCAA & GGCCAGCACGTGAAGAAGAT \\
\hline
\end{tabular}

$h$ Human 


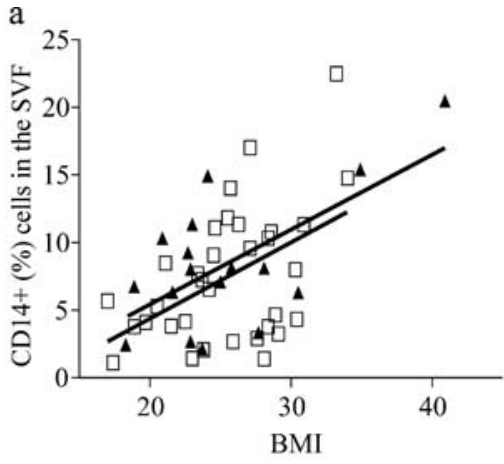

b

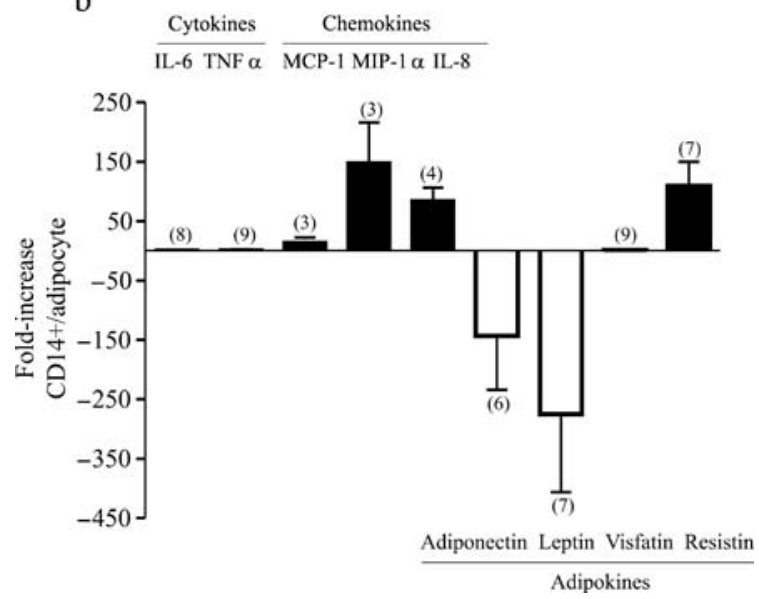

Fig. 1 Visceral white adipose tissue macrophages. a Correlation between $\mathrm{CD}_{14}{ }^{+}$cells in visceral white adipose tissue and BMI (squares men, $r=0.3702, p=0.017$; triangles women, $r=0.3507$, $p=0.083)$. SVF stroma-vascular fraction. b Expression of IL-6, TNF- $\alpha$, MCP-1, MIP-1 $\alpha$, IL-8, leptin, adiponectin, visfatin and resistin. Data, normalised to $18 \mathrm{~S}$ RNA levels, are expressed as foldincrease in $\mathrm{CD} 14^{+}$cells compared with adipocytes from $(n)$ tissues $($ mean \pm SEM)

Cytokine, chemokine and adipokine expression in $\mathrm{CD} 14^{+}$cells and adipocytes

Real-time PCR analyses were performed on freshly isolated $\mathrm{CD} 14^{+}$cells and mature adipocytes. As depicted in Fig. 1b, both cell types expressed IL- 6 and TNF- $\alpha$, although the level of TNF- $\alpha$ mRNA was very low (on average 277-fold less than that of IL-6 transcripts). The chemokines MCP-1, MIP- $1 \alpha$ and IL-8 were mainly expressed by $\mathrm{CD} 14^{+}$cells, whereas leptin and adiponectin were exclusively identified in mature adipocytes. Surprisingly, visfatin mRNAs, identified in adipocytes, was also found in $\mathrm{CD}_{14}{ }^{+}$cells, while resistin transcripts were predominantly expressed in $\mathrm{CD} 14^{+}$cells.

Effects of $\mathrm{CD} 14^{+}$cell- and adipocyte-derived secretory products on human hepatocytes

The determination of the visfatin and resistin concentrations in conditioned media from $\mathrm{CD} 14^{+}$cells and mature adipocytes confirmed the predominant production of both adipokines by $\mathrm{CD} 14^{+}$cells (Fig. 2a). Treatment of HepG2 cells for $3 \mathrm{~min}$ with $\mathrm{CD}^{+} 4^{+}$cell-derived secretions increased phosphorylation levels of protein kinase B (Akt) (Fig. 2b), whereas conditioned media from adipocytes enhanced the phosphorylation of extracellular signalregulated kinase 1/2 (Fig. 2c). The level of phosphorylation of AMP-activated protein kinase remained unchanged under the same conditions (data not shown).
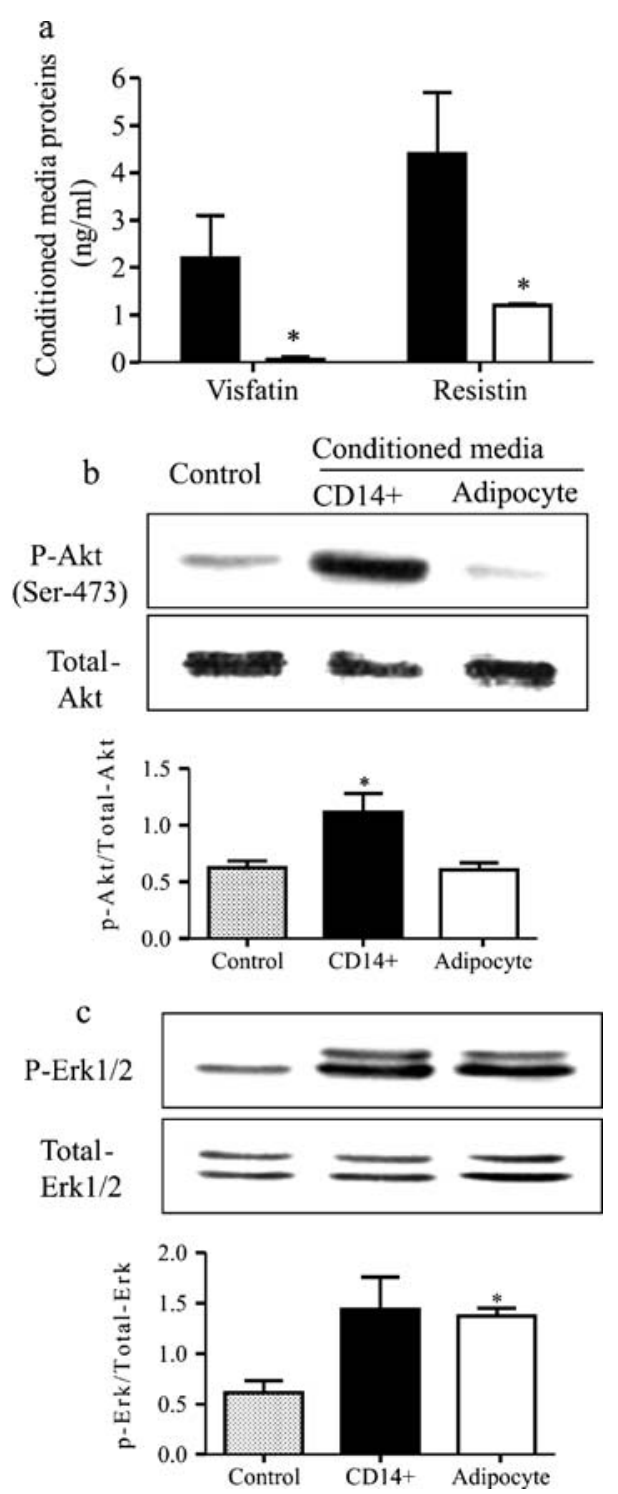

Fig. 2 Secretions of $\mathrm{CD} 14^{+}$cells and adipocytes. a Visfatin and resistin concentrations in conditioned media from $\mathrm{CD} 14^{+}$cells (filled bars) and adipocytes (open bars). Results are mean $\pm \mathrm{SEM}$, $n=4, * p<0.05$. b Effects of conditioned media on phosphorylated Akt (Ser-473) in HepG2 cells. Values are mean \pm SEM, $n=4$, $* p<0.05$. c Effects of conditioned media on phosphorylation of extracellular signal-regulated kinase (Erk) $1 / 2$ in HepG2 cells. Values are mean \pm SEM, $n=3$ for $\mathrm{CD} 14^{+}$cells and $n=4$ for adipocytes, $* p<0.05$ 


\section{Discussion}

Obesity is considered a low-inflammatory condition. An increasing number of reports suggest that the adipose tissue itself might be a source of proinflammatory factors and a target of inflammatory processes [5]. The present study shows that human visceral WAT, pathophysiologically the most relevant fat mass depot, contains a population of $\mathrm{CD} 14^{+}$cells the proportion of which increases with BMI. We demonstrated previously that cells positive for the CD14 marker, present in the SVF of human subcutaneous WAT, exhibited the typical macrophage phenotype and activity [4]. Moreover, we showed that the $\mathrm{CD} 14^{+}$cells were unable to differentiate into adipocytes under adipogenic culture conditions [6], demonstrating that the CD14 cells did not contain preadipocyte-like cells. Finally, the present FACS analysis data confirmed the coexpression by the $\mathrm{CD} 14^{+}$cell population of two other markers, CD45 and CD31, which are hallmarks of macrophages. Thus, the CD14 cell marker allows the identification of adipose tissue-derived macrophages. This is in contrast to the other widely used macrophage marker, CD68, recently shown to be also expressed by human preadipocytes and upregulated in obese subjects [7]. Further analysis of the human visceral WAT macrophage phenotype showed that the cells expressed higher levels of the chemokines MCP-1, MIP$1 \alpha$ and IL-8 than adipocytes. The two proinflammatory cytokines TNF- $\alpha$ and IL- 6 were both expressed equally in macrophages and adipocytes. Induction of expression of proinflammatory factors, particularly $\mathrm{TNF}-\alpha$, has been reported to occur following collagenase digestion of murine adipose cells [8]. However, in the present study only low levels of TNF- $\alpha$ transcripts were detected in both cell types. It is thus unlikely that the distinct transcript distribution patterns in adipocytes and macrophages are related to the isolation procedure. Surprisingly, resistin and visfatin were found to be predominantly produced and released by the WAT-derived macrophages. The adipokines resistin and visfatin have been implicated in the modulation of insulin signalling in the liver in rodents [9, 10]. However, the physiological relevance of resistin in man is under debate. Indeed, human adipocytes lack resistin expression, whereas adipocytes are the main source of resistin production in rodents. Recent reports have shown induction of resistin expression in human blood monocytes that have differentiated into macrophages [11], as well as an increased resistin concentration in atherosclerosis [12]. Moreover, visfatin is also considered to be an inflammatory cytokine (pre-B cell colony-enhancing factor) in neutrophils [13]. It is suggested that resistin and visfatin in the visceral WAT might be considered to be proinflammatory markers. Finally, visceral WAT macrophages, but not adipocyte-derived products, led to the stimulation of Akt in human hepatocytes. Although additional experiments are required to determine the macrophage-derived factor(s) responsible for such an effect, resistin and visfatin might be good candidates since both have been described to activate Akt in hepatocytes $[9,10]$. Taken together, the results demonstrate that the visceral WAT develops a proinflammatory state in obesity and suggest that the accumulation of macrophages might be responsible for the enhanced fat mass-derived production of chemokines as well as resistin and visfatin in obesity.

Acknowledgements We acknowledge grants from the Alexander von Humboldt Foundation and Sofja Kovalevskaja Price (Humboldt Foundation and the German Federal Ministry of Education and Research).

\section{References}

1. Lebovitz HE (2003) The relationship of obesity to the metabolic syndrome. Int J Clin Pract Suppl:18-27

2. Trayhurn P, Wood IS (2004) Adipokines: inflammation and the pleiotropic role of white adipose tissue. Br J Nutr 92:347-355

3. Weisberg SP, McCann D, Desai M, Rosenbaum M, Leibel RL, Ferrante AW Jr (2003) Obesity is associated with macrophage accumulation in adipose tissue. J Clin Invest 112:1796-1808

4. Curat CA, Miranville A, Sengenes C et al (2004) From blood monocytes to adipose tissue-resident macrophages: induction of diapedesis by human mature adipocytes. Diabetes 53:1285-1292

5. Bouloumie A, Curat CA, Sengenes C, Lolmede K, Miranville A, Busse R (2005) Role of macrophage tissue infiltration in metabolic diseases. Curr Opin Clin Nutr Metab Care 8:347-354

6. Sengenes C, Lolmede K, Zakaroff-Girard A, Busse R, Bouloumie A (2005) Preadipocytes in the human subcutaneous adipose tissue display distinct features from the adult mesenchymal and hematopoietic stem cells. J Cell Physiol 205:114-122

7. Nair S, Lee YH, Rousseau E et al (2005) Increased expression of inflammation-related genes in cultured preadipocytes/stromal vascular cells from obese compared with non-obese Pima Indians. Diabetologia 48:1784-1788

8. Ruan H, Zarnowski MJ, Cushman SW, Lodish HF (2003) Standard isolation of primary adipose cells from mouse epididymal fat pads induces inflammatory mediators and downregulates adipocyte genes. J Biol Chem 278:47585-47593

9. Muse ED, Obici S, Bhanot S et al (2004) Role of resistin in dietinduced hepatic insulin resistance. J Clin Invest 114:232-239

10. Fukuhara A, Matsuda M, Nishizawa M et al (2005) Visfatin: a protein secreted by visceral fat that mimics the effects of insulin. Science 307:426-430

11. Lehrke M, Reilly MP, Millington SC, Iqbal N, Rader DJ, Lazar MA (2004) An inflammatory cascade leading to hyperresistinemia in humans. PLoS Med 1:e45

12. Reilly MP, Lehrke M, Wolfe ML, Rohatgi A, Lazar MA, Rader DJ (2005) Resistin is an inflammatory marker of atherosclerosis in humans. Circulation 111:932-939

13. Jia SH, Li Y, Parodo J et al (2004) Pre-B cell colony-enhancing factor inhibits neutrophil apoptosis in experimental inflammation and clinical sepsis. J Clin Invest 113:1318-1327 